\title{
Rotation measure variations for 20 millisecond pulsars
}

\author{
W. M. $\operatorname{Yan}^{1,2,3}$ • R. N. Manchester ${ }^{3}$ - G. Hobbs ${ }^{3}$ • \\ W. van Straten ${ }^{4}$ - J. E. Reynolds ${ }^{3}$ - N. Wang ${ }^{1,5}$ • \\ M. Bailes ${ }^{4}$ • N. D. R. Bhat ${ }^{4}$ • S. Burke-Spolaor ${ }^{3}$ • \\ D. J. Champion ${ }^{6}$ - A. Chaudhary ${ }^{3}$ - W. A. Coles $^{7}$ \\ - A. W. Hotan ${ }^{3}$ - J. Khoo ${ }^{3}$ - S. Oslowski ${ }^{4,3}$ • \\ J. M. Sarkissian ${ }^{3}$ •D. R. B. Yardley ${ }^{8,3}$
}

\begin{abstract}
We report on variations in the mean position angle of the 20 millisecond pulsars being observed as part of the Parkes Pulsar Timing Array (PPTA)
\end{abstract}
W. M. Yan
R. N. Manchester
G. Hobbs
W. van Straten
J. E. Reynolds
N. Wang
M. Bailes
N. D. R. Bhat
S. Burke-Spolaor
D. J. Champion
A. Chaudhary
W. A. Coles
A. W. Hotan
J. Khoo
S. Oslowski
J. M. Sarkissian
D. R. B. Yardley

${ }^{1}$ Xinjiang Astronomical Observatory, Chinese Academy of Sciences, 150 Science-1 Street, Urumqi, Xinjiang 830011, China yanwm@uao.ac.cn

${ }^{2}$ Graduate University of Chinese Academy of Sciences, 19A Yuquan Road, Beijing 100049, China

${ }^{3}$ CSIRO Astronomy and Space Science, Australia Telescope National Facility, PO Box 76, Epping NSW 1710, Australia

${ }^{4}$ Centre for Astrophysics and Supercomputing, Swinburne University of Technology, PO Box 218, Hawthorn VIC 3122, Australia

${ }^{5}$ Key Laboratory of Radio Astronomy, Chinese Academy of Sciences Nanjing, 210008, China

${ }^{6}$ Max-Planck-Institut für Radioastronomie, Auf dem Hügel 69, 53121 Bonn, Germany

${ }^{7}$ Electrical and Computer Engineering, University of California at San Diego, La Jolla, California, USA

${ }^{8}$ Sydney Institute for Astronomy, School of Physics, The University of Sydney, NSW 2006, Australia project. It is found that the observed variations are dominated by changes in the Faraday rotation occurring in the Earth's ionosphere. Two ionospheric models are used to correct for the ionospheric contribution and it is found that one based on the International Reference Ionosphere gave the best results. Little or no significant long-term variation in interstellar RM was found with limits typically about $0.1 \mathrm{rad} \mathrm{m}^{-2} \mathrm{yr}^{-1}$ in absolute value. In a few cases, apparently significant RM variations over timescales of a few 100 days or more were seen. These are unlikely to be due to localised magnetised regions crossing the line of sight since the implied magnetic fields are too high. Most probably they are statistical fluctuations due to random spatial and temporal variations in the interstellar electron density and magnetic field along the line of sight.

Keywords pulsars: general — ISM: general — radio continuum: stars

\section{Introduction}

The observed radiation from pulsars is affected by propagation effects along the path between the pulsar and the observatory. One of the various propagation effects is Faraday rotation, that is, the rotation of the plane of linear polarization caused by the different phase velocities of the two hands of circular polarization in a magnetised plasma. Pulsar radiation typically has strong linear polarization making it relatively easy to observe Faraday rotation for these sources. Faraday rotation is described by the rotation measure (RM), defined by

$\psi=\mathrm{RM} \lambda^{2}$,

where $\psi$ is the linear polarization position angle (PA) and $\lambda=c / \nu$ is the radio wavelength corresponding to 
radio frequency $\nu$. The rotation measure is approximated by

$\mathrm{RM}=0.810 \int_{0}^{D} n_{e} \boldsymbol{B} \cdot d \boldsymbol{l}$,

where $n_{e}$ is the interstellar electron density in units of $\mathrm{cm}^{-3}, \boldsymbol{B}$ is the vector magnetic field in microgauss, $d \boldsymbol{l}$ is an elemental vector along the line-of-sight toward us (positive RMs correspond to fields directed towards us) in parsecs and $\mathrm{RM}$ is in units of $\mathrm{rad} \mathrm{m}^{-2}$.

Along with the dispersion measure (DM), defined by

$\mathrm{DM}=\int_{0}^{D} n_{e} d l$,

where DM is in units of $\mathrm{cm}^{-3} \mathrm{pc}$, the RM may be used to determine the strength and direction of the interstellar magnetic field (see, e.g., Manchester 1974; Han et al. 2006; Noutsos et al. 2008). Combining Equations 2 and 3, we obtain an estimate of the mean line-of-sight component of the interstellar magnetic field weighted by the local electron density along the path:

$\left\langle B_{\|}\right\rangle=1.232 \frac{\mathrm{RM}}{\mathrm{DM}} \mu \mathrm{G}$,

where RM and DM are in their usual units.

Long-term changes in pulsar PAs can result from changes in the polarization of the emitted radiation or from changes in the RM along the path (Equation (10). Intrinsic PA changes could result from precession of the pulsar spin axis or changes in the magnetic configuration of the star. RM changes can occur as the path to the pulsar traverses different regions of the interstellar medium (ISM) or in the Earth's ionosphere due to the diurnal and other changes in the ionospheric total electron content.

Correlated RM and DM variations with time have been observed for several pulsars. Hamilton et al. (1977, 1985) analyze the variations of RM and DM for the Vela pulsar, finding an approximately linear variation in both with slopes of $0.73 \mathrm{rad} \mathrm{m}^{-2} \mathrm{yr}^{-1}$ and $-0.04 \mathrm{~cm}^{-3} \mathrm{pc} \mathrm{yr}^{-1}$ for RM and DM respectively. They interpret the RM and DM variations as the result of the relative motion of a magnetized filament of the Vela supernova remnant across the line-of-sight and estimated the electron density and the effective mean line-of-sight component of magnetic field in the filament. Adopting $500 \mathrm{~km} \mathrm{~s}^{-1}$ as the transverse velocity of the source, they obtain a transverse scale length of $5.1 \times 10^{-4} \mathrm{pc}$ per year. Assuming that the line-of-sight dimension is comparable with the transverse scale gives an electron density in the region of $\Delta n_{e}=78 \mathrm{~cm}^{-3}$. The mean line-of-sight component of magnetic field in the varying region is given by

$\left\langle B_{\|}\right\rangle_{\mathrm{var}}=1.23(d \mathrm{RM} / d t) /(d \mathrm{DM} / d t) \mu \mathrm{G}$

(cf. Equation 4) and for Vela is $22 \mu \mathrm{G}$.

van Ommen et al. (1997) made a similar analysis for PSRs B1556-44 and B1727-47. For PSR B1556-44, the estimated electron density and the effective mean line-of-sight component of magnetic field in the varying region are $\Delta n_{e}=290 \mathrm{~cm}^{-3}$ and $\left\langle B_{\|}\right\rangle_{\mathrm{var}}=2 \mu \mathrm{G}$ directed away from the pulsar; for PSR B1727-47, the electron density is $\Delta n_{e}=170 \mathrm{~cm}^{-3}$, the magnetic field strength is $\left\langle B_{\|}\right\rangle_{\text {var }}=16 \mu \mathrm{G}$ directed toward the pulsar. Because of the absence of an associated supernova remnant the authors proposed that the RM variation for PSR B1727-47 may be due to a an irregularity within the HII region of I Scorpius located at a distance of $1.3 \mathrm{kpc}$. Rankin et al. (1988) made a detailed analysis of the RM and DM variations for the Crab pulsar and found correlated variations between 1972 and 1974 which imply a magnetic field in the varying region of about $170 \mu \mathrm{G}$. Several other authors (e.g. Weisberg et al. 2004; Han et al. 2006) also find apparent time variations in the interstellar RMs of several pulsars.

Much stronger fields are observed in vicinity of stars. For example, Johnston et al. (2005) observed RM variations of many $1000 \mathrm{rad} \mathrm{m}^{-2}$ and DM variations of $\sim 20 \mathrm{~cm}^{-3} \mathrm{pc}$ for signals propagating through the disk of SS 2883, the companion star to PSR B1259-63, implying fields in the $\mathrm{mG}$ range. $\mathrm{RM}$ variations of a few $100 \mathrm{rad} \mathrm{m}^{-2}$ have been observed as the path to PSR J1801-2304 passed close to the Sun (Ord et al. 2007). With expected values of electron density in the solar corona, these variations again imply fields of order $1 \mathrm{mG}$.

The Parkes Pulsar Timing Array (PPTA) project is making regular timing observations of 20 millisecond pulsars (MSPs) with the primary goal of detecting lowfrequency $(\mathrm{nHz})$ gravitational waves (Manchester 2008; Verbiest et al. 2010). Data from the first three years of this project $(2004-2007)$ were used by You et al. (2007) to investigate the DM variations in the PPTA pulsars. Significant variations were found in many of the pulsars, with observed rates of change up to $\sim 10^{-2} \mathrm{pc} \mathrm{cm}^{-3} \mathrm{yr}^{-1}$. The PPTA sample is unusual if not unique in having a large number of observations over a long data span with high signal/noise ratio and accurately calibrated polarization data for a significant number of pulsars. Mean pulse total intensity and polarization profiles have been published for the 20 PPTA pulsars by Yan et al. (2011) showing that the pulsed 
emission extends over more than half of the pulse period for most of the sample and that the polarization variations across the profile are generally complex.

In this paper we report on the long-term variations of observed mean PAs over a data span of 4.8 years for the 20 PPTA MSPs. It is shown that, after correcting for Faraday rotation in the Earth's ionosphere, there is little or no significant variation in the observed PAs and hence also in the interstellar RMs. Details of the observations and data processing are given in 92 a comparison between two different ionospheric RM correction methods is made in $\$ 3$, the observed variations are discussed in 4 and we provide a short summary in 95 .

\section{Observations and analysis}

The PPTA observations were made at intervals of 2 - 3 weeks using the Parkes $64-\mathrm{m}$ radio telescope with the centre beam of the $20 \mathrm{~cm}$ Multibeam receiver (Staveley-Smith et al. 1996). The Multibeam receiver has a bandwith of about $300 \mathrm{MHz}$ centered at about $1.4 \mathrm{GHz}$. The system equivalent flux density on cold sky is approximately 30 Jy. The Multibeam receiver has an orthogonal linearly polarized feed system. There is a calibration probe at $45^{\circ}$ to the two signal probes through which a linearly polarized broadband and pulsed calibration signal can be injected. Three different back-end systems were used, namely the Parkes digital filterbank systems PDFB1, PDFB2 and PDFB4. The PDFB systems are digital polyphase filterbanks using field-programmable gate array (FPGA) processors. Signals from each of the two orthogonal polarizations are band-limited to $256 \mathrm{MHz}$ and 8-bit sampled at the Nyquist rate. After transformation to the spectral domain, typically with 1024 channels across the band, data are folded at the apparent pulse period to form mean pulse profiles in the four Stokes parameters.

All data were recorded using the PSRFITS data format (Hotan et al. 2004) with 1-minute sub-integrations. For PSRs J1857+0943, J1939+2134 and J2124-3358, each observation was 32 minutes duration; for the other 17 pulsars the observation times were 64 minutes. A short 2-minute observation of the pulsed calibration signal was made before each pulsar observation. Flux density scales were established using observations of Hydra A which was assumed to have a flux density of $43.1 \mathrm{Jy}$ at $1400 \mathrm{MHz}$ and a spectral index of -0.91 . The Multibeam receiver has significant coupling between the two signal feed probes. The cross-coupling was measured by analysis of series of observations of PSR J0437-4715 covering a wide range of parallactic angles (van Straten 2004).
All observations were processed using the PSRCHIVE software package (Hotan et al. 2004). Strong narrowband radio frequency interference (RFI) was identified by searching for spectral channels that were significantly above the local mean; such channels were excised from the data. Similarly, broad-band impulsive RFI was identified on zero-DM summed time-domain data and excised. The variations in instrumental gain and phase across the band and the effects of crosscouping in the feed were removed using the calibration observations. Polarization parameters are in accordance with the astronomical conventions described by van Straten et al. (2010); in particular, PAs are absolute and referred to celestial north, increasing toward east. Table1 1ists the basic parameters for the 20 PPTA MSPs with RM values derived from PDFB2 observations following Yan et al. (2011).

To measure the long-term changes in mean PA, we took the weighted average of the differences between the PAs across an individual observation profile and the PAs across the grand-average profile from Yan et al. (2011). This gives a relative PA shift for that observation and its estimated uncertainty. This is then repeated for all observations of a given pulsar to give the $\mathrm{PA}$ variations across the data span. In order to accurately align the observation profiles for a given pulsar, pulse times of arrival were obtained for all the observations and fitted using the TEMPO2 pulsar timing package (Hobbs et al. 2006) to give a timing model for the pulsar. This timing model was then used to align the profiles before computing the PA differences.

Because the Earth's magnetic field is relatively strong ( $0.5 \mathrm{G})$ and the electron density in the Earth's ionosphere relatively high (up to $10^{6} \mathrm{~cm}^{-3}$ ), the Earth's ionosphere makes a signficant contribution to the total RM along the path to the pulsar. For the Southern Hemisphere, the Earth's magnetic field is directed outwards and so the ionospheric contribution to the RM is negative, typically between -0.5 and $-3 \mathrm{rad} \mathrm{m}^{-2}$, with a strong diurnal variation due to the varying ionspheric electron density.

\section{Ionospheric RM corrections}

We have used two programs to compute the ionospheric $\mathrm{RM}(\mathrm{RMi})$ in the direction of a given source at a given time. The first, FARRot, was developed at the Dominion Radio Astrophysical Observatory (DRAO), Penticton, Canada. It uses three Chapman layers to model the $\mathrm{D}, \mathrm{E}$ and $\mathrm{F}$ regions and a semi-empirical model for variations in these based on the $10.7-\mathrm{cm}$ solar radio flux measured by DRAO. The second, GETRM_IONO, 
Table 1 Parameters for the 20 PPTA pulsars

\begin{tabular}{|c|c|c|c|c|c|}
\hline PSR & $\begin{array}{c}P \\
(\mathrm{~ms}) \\
\end{array}$ & $\begin{array}{c}\mathrm{DM} \\
\left(\mathrm{cm}^{-3} \mathrm{pc}\right) \\
\end{array}$ & $\begin{array}{c}\mathrm{RM} \\
\left(\mathrm{rad} \mathrm{m}^{-2}\right) \\
\end{array}$ & $\begin{array}{c}\text { Data span } \\
(\mathrm{MJD})\end{array}$ & $\begin{array}{l}\mathrm{Nr} \text { of } \\
\text { Obs. }\end{array}$ \\
\hline J0437-4715 & 5.757 & 2.64 & -0.6 & $53703-55312$ & 393 \\
\hline J0613-0200 & 3.062 & 38.78 & 9.7 & $53687-55407$ & 125 \\
\hline J0711-6830 & 5.491 & 18.41 & 21.4 & $53660-55408$ & 47 \\
\hline $\mathrm{J} 1022+1001$ & 16.453 & 10.25 & -0.3 & $53727-55406$ & 84 \\
\hline J1024-0719 & 5.162 & 6.49 & -8.3 & $53688-55407$ & 79 \\
\hline J1045-4509 & 7.474 & 58.17 & 92.3 & $53660-55408$ & 110 \\
\hline J1600-3053 & 3.598 & 52.33 & -15.8 & $53660-55406$ & 103 \\
\hline J1603-7202 & 14.842 & 38.05 & 27.7 & $53703-55406$ & 101 \\
\hline J1643-1224 & 4.622 & 62.41 & -308.2 & $53724-55406$ & 98 \\
\hline $\mathrm{J} 1713+0747$ & 4.570 & 15.99 & 9.3 & $53660-55406$ & 112 \\
\hline J1730-2304 & 8.123 & 9.62 & -7.2 & $53725-55387$ & 70 \\
\hline J1732-5049 & 5.313 & 56.82 & -6.7 & $53799-55117$ & 22 \\
\hline J1744-1134 & 4.075 & 3.14 & -1.6 & $53660-55406$ & 107 \\
\hline J1824-2452 & 3.054 & 120.50 & 77.6 & $53688-55350$ & 76 \\
\hline $\mathrm{J} 1857+0943$ & 5.362 & 13.30 & 18.2 & $53703-55406$ & 62 \\
\hline J1909-3744 & 2.947 & 10.39 & -6.5 & $53688-55406$ & 174 \\
\hline $\mathrm{J} 1939+2134$ & 1.558 & 71.04 & 6.9 & $53816-55406$ & 76 \\
\hline $\mathrm{J} 2124-3358$ & 4.931 & 4.60 & -5.4 & $53660-55406$ & 103 \\
\hline J2129-5721 & 3.726 & 31.85 & 23.5 & $53724-55389$ & 62 \\
\hline $\mathrm{J} 2145-0750$ & 16.052 & 9.00 & -1.3 & $53725-55389$ & 67 \\
\hline
\end{tabular}

was originally written by J. L. Han for correction of measured pulsar RMs (e.g., Han et al. 2006). In its current form, it uses the 2007 International Reference Ionosphere (IRI) mode 1 with the URSI model for the critical frequency of the $\mathrm{F} 2$ ionospheric layer $\left(f_{0} \mathrm{~F} 2\right)$ and input of the geomagnetic Ap index, the solar 10.7-cm radio flux and sunspot numbers. Both models use the International Geomagnetic Reference Field 2 to define the structure of the Earth's magnetic field.

We have used these programs to compute RMi for each observation of each pulsar. Observed PA differences have been corrected for the rotation occurring in the ionosphere to give the PAs above the ionosphere. In Figure 1-4 we show the observed PA differences in the upper panel for the 20 PPTA pulsars. The middle panel gives the PA differences after correction for ionospheric rotation using FARROT to compute RMi and the lower panel shows the PA differences after correction using GETRM_IONO.

This figure shows that the PA variations are less after removal of the ionospheric contribution. This is especially noticable for pulsars in the northern sky, e.g.,

${ }^{1}$ See http://iri.gsfc.nasa.gov for a general description of the IRI. Source and data files are available from http://nssdcftp.gsfc.nasa.gov/models/ionospheric/iri/iri2007/

${ }^{2}$ See http://www.ngdc.noaa.gov/IAGA/vmod/igrf.html
PSRs J1713+0747 and J1939+2134. For these pulsars viewed from Parkes the line of sight to the pulsar is roughly tangential to the local magnetic field (the dip angle at Parkes is about $-60^{\circ}$ ) and so the RMi is large. There is a clear annual variation in the observed PAs resulting from the aliasing of the diurnal ionospheric variation with the annual sidereal motion. This annual variation is largely removed by the ionospheric correction. Table 2 gives the rms scatter in the PAs and the corresponding reduced $\chi^{2}$ after a weighted fit of a straight line to the corrected PAs for both the FARROT and IRI corrections for the PPTA pulsars. Without exception the PAs after the IRI corrections have a smaller rms scatter and reduced $\chi^{2}$ than those after the FARROT correction.

\section{Results and Discussion}

After the ionospheric correction, the PA variations for essentially all of the PPTA pulsars are dominated by a short-term scatter. As shown by the large values of reduced $\chi^{2}$ (Table 2), this scatter is much larger than the nominal uncertainty of the PAs in most cases. For the stronger pulsars, the PA uncertainty estimated from the off-pulse rms variations in the Stokes profiles is very 

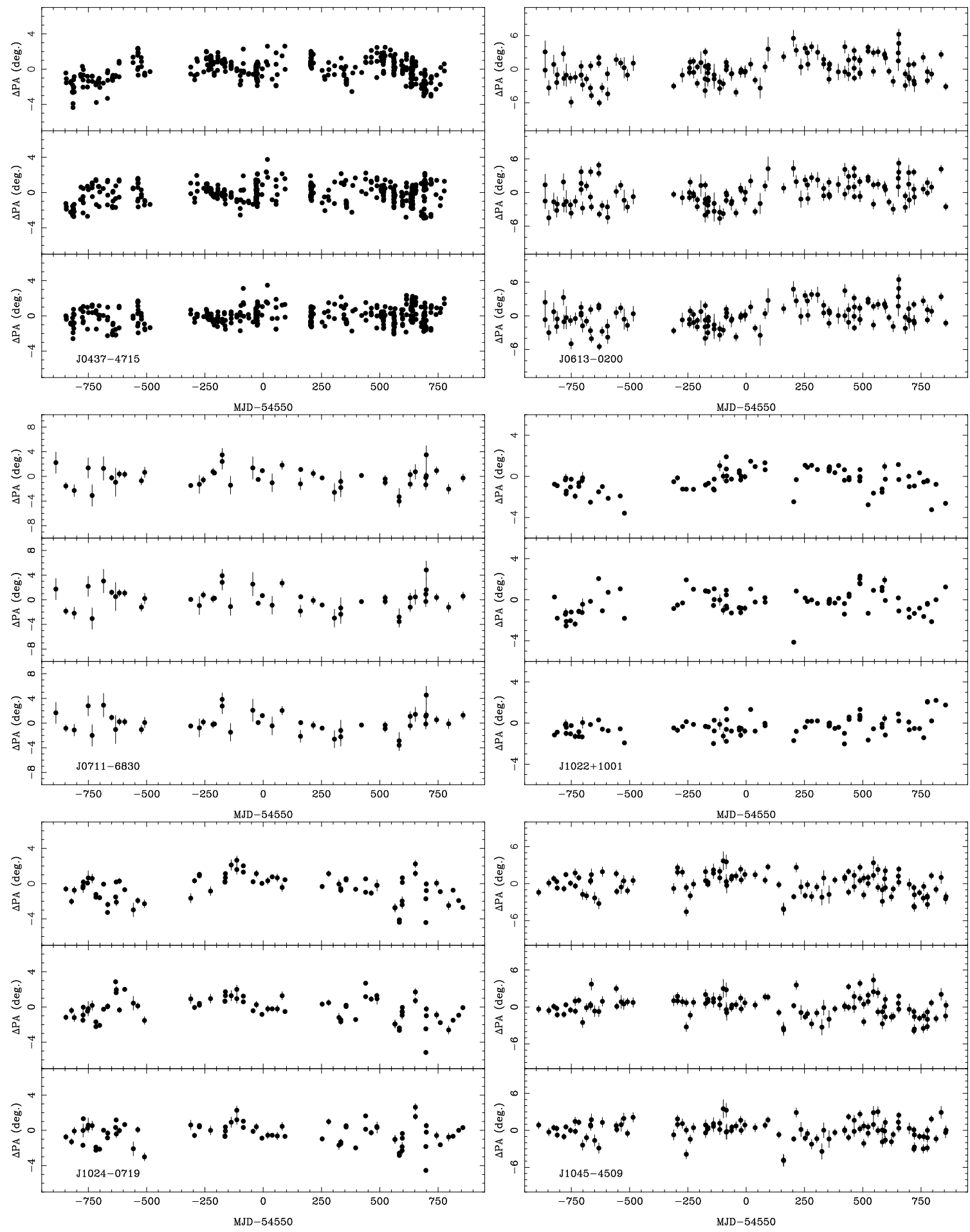

Fig. 1 PA differences as a function of time for six of the PPTA pulsars. In each sub-figure, the upper panel shows PA differences without ionospheric correction, the middle panel gives PA differences after ionospheric corrections using the Penticton program FARROT; the lower part shows differences after ionospheric corrections using the IRI ionospheric model. 

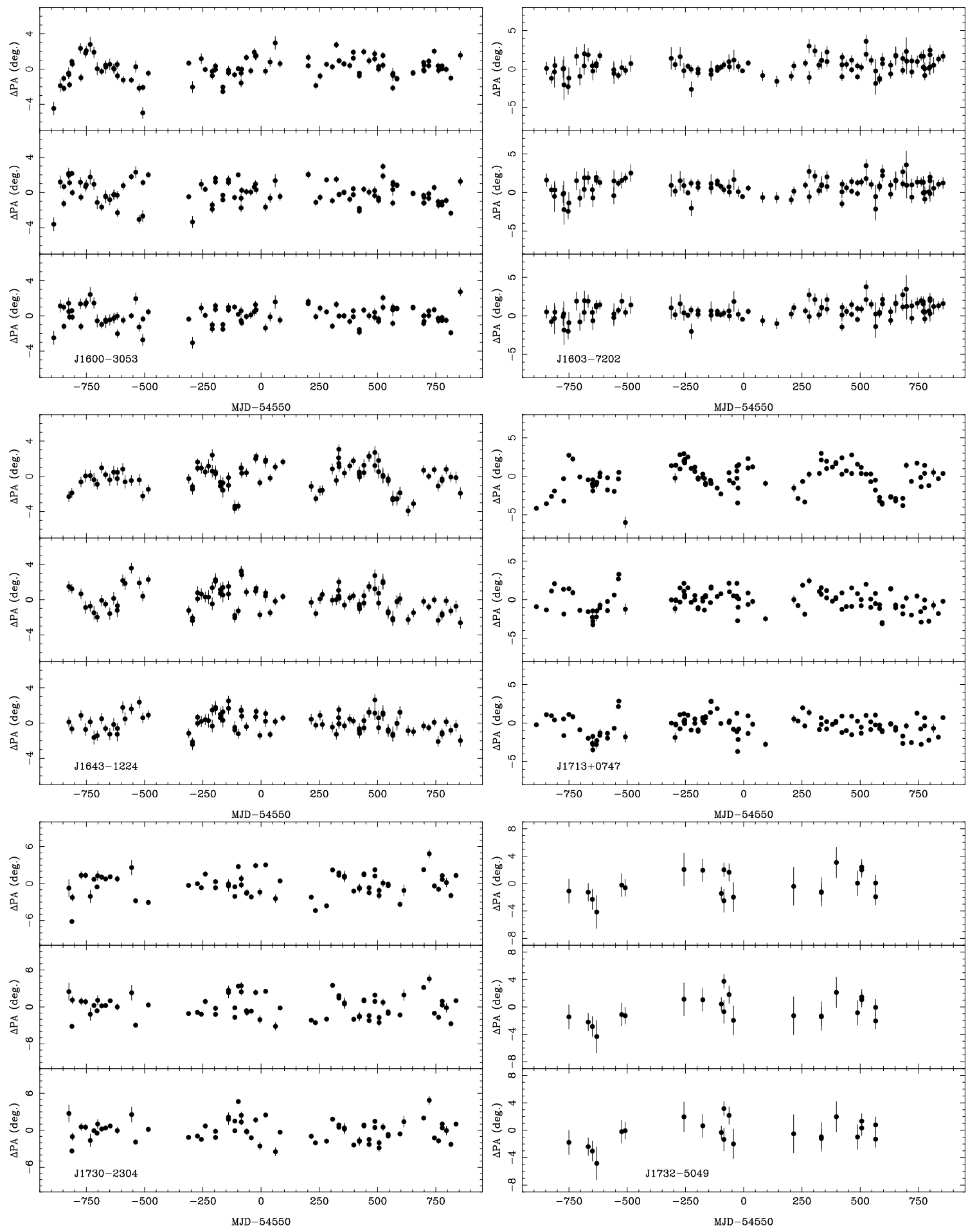

Fig. 2 PA differences as a function of time for six of the PPTA pulsars. See Figure 1 for details. 

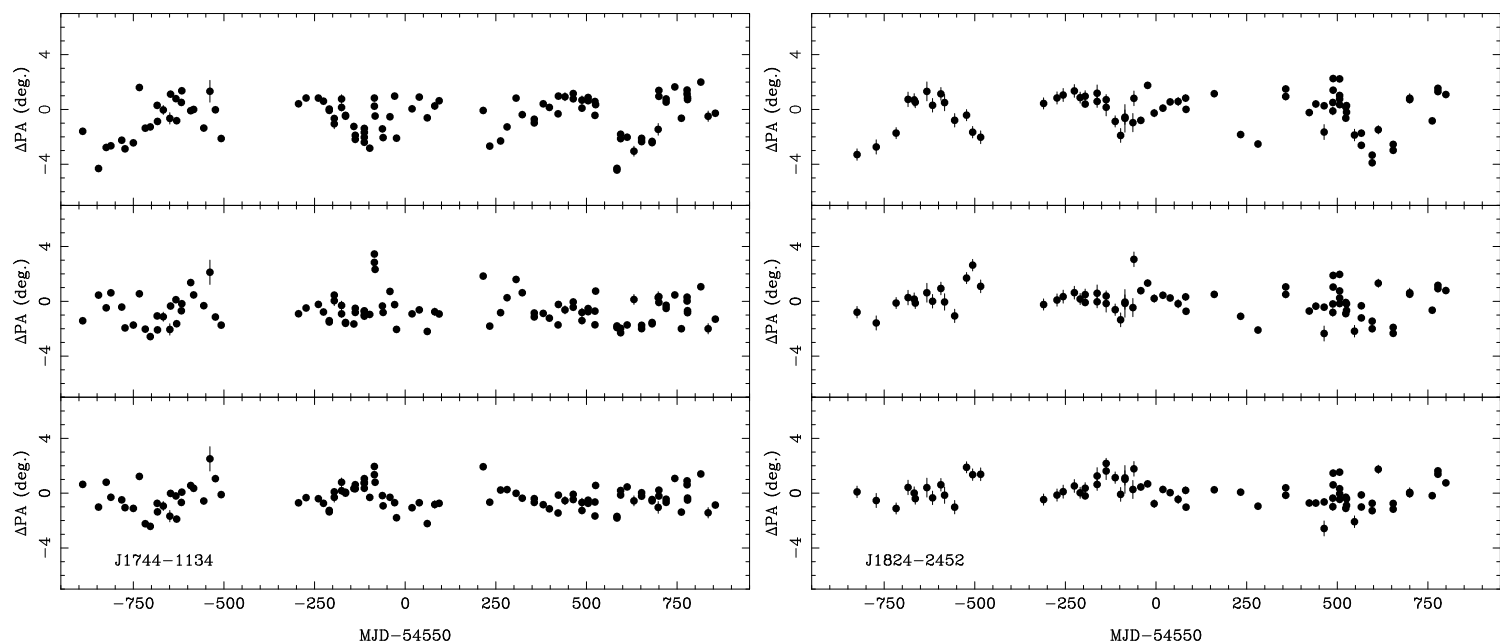

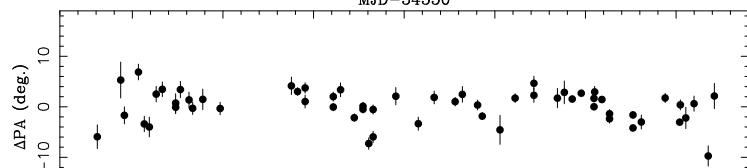

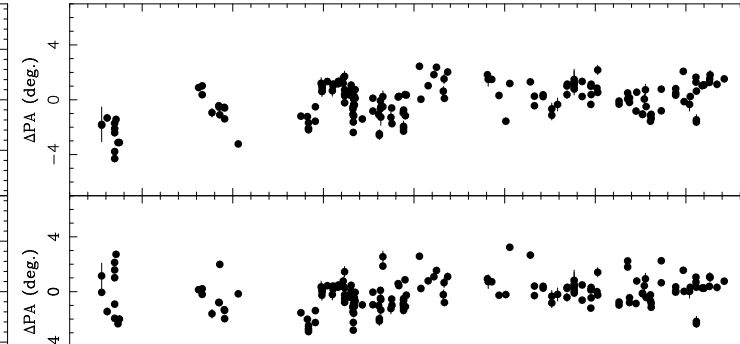

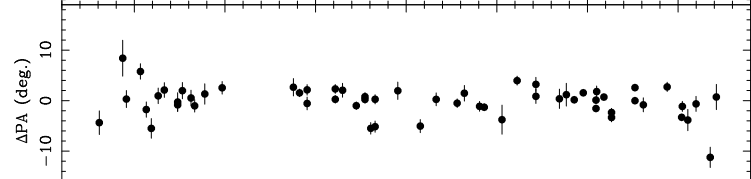

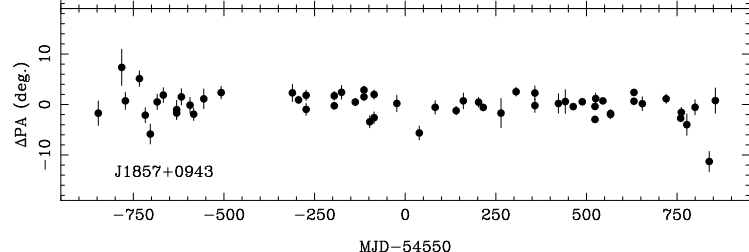

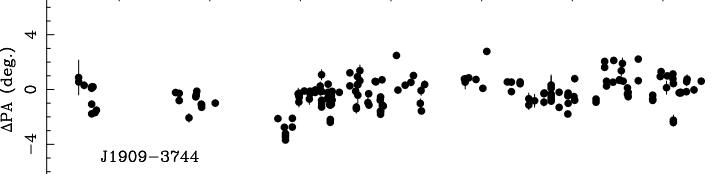
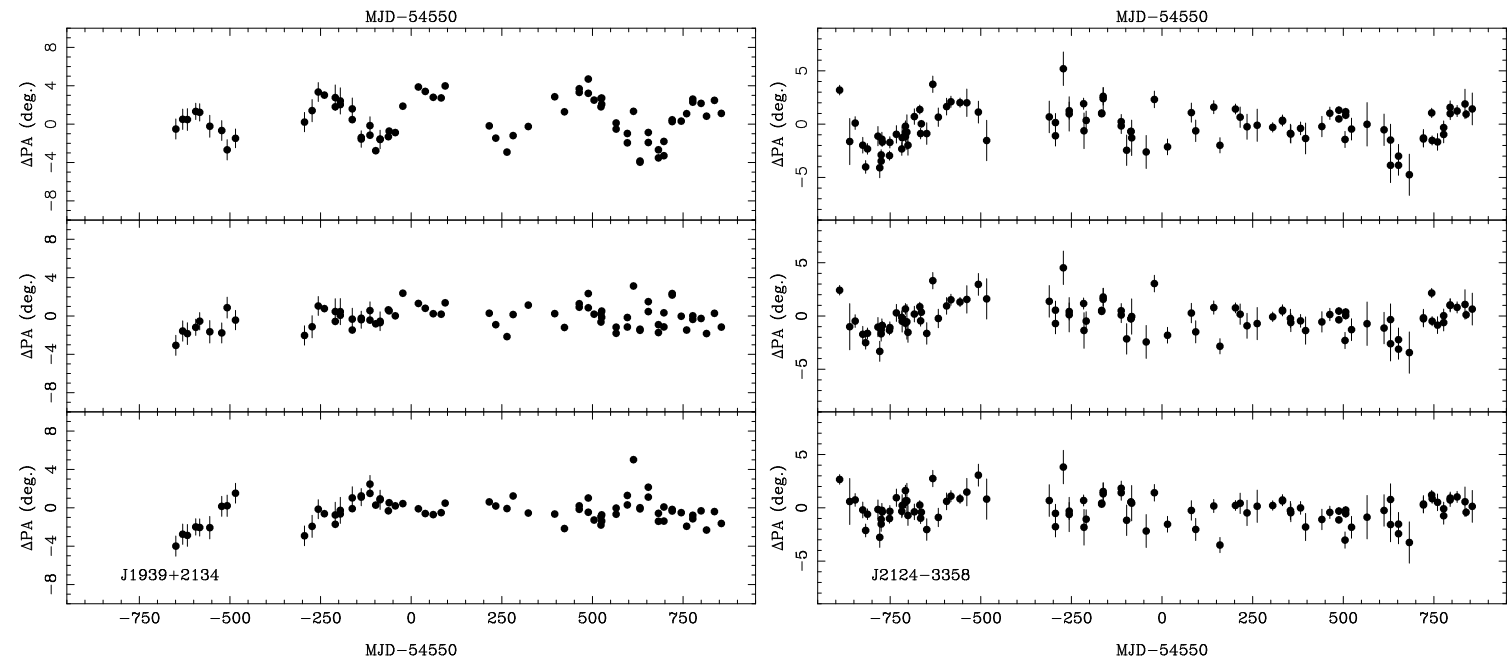

Fig. 3 PA differences as a function of time for six of the PPTA pulsars. See Figure 1 for details. 

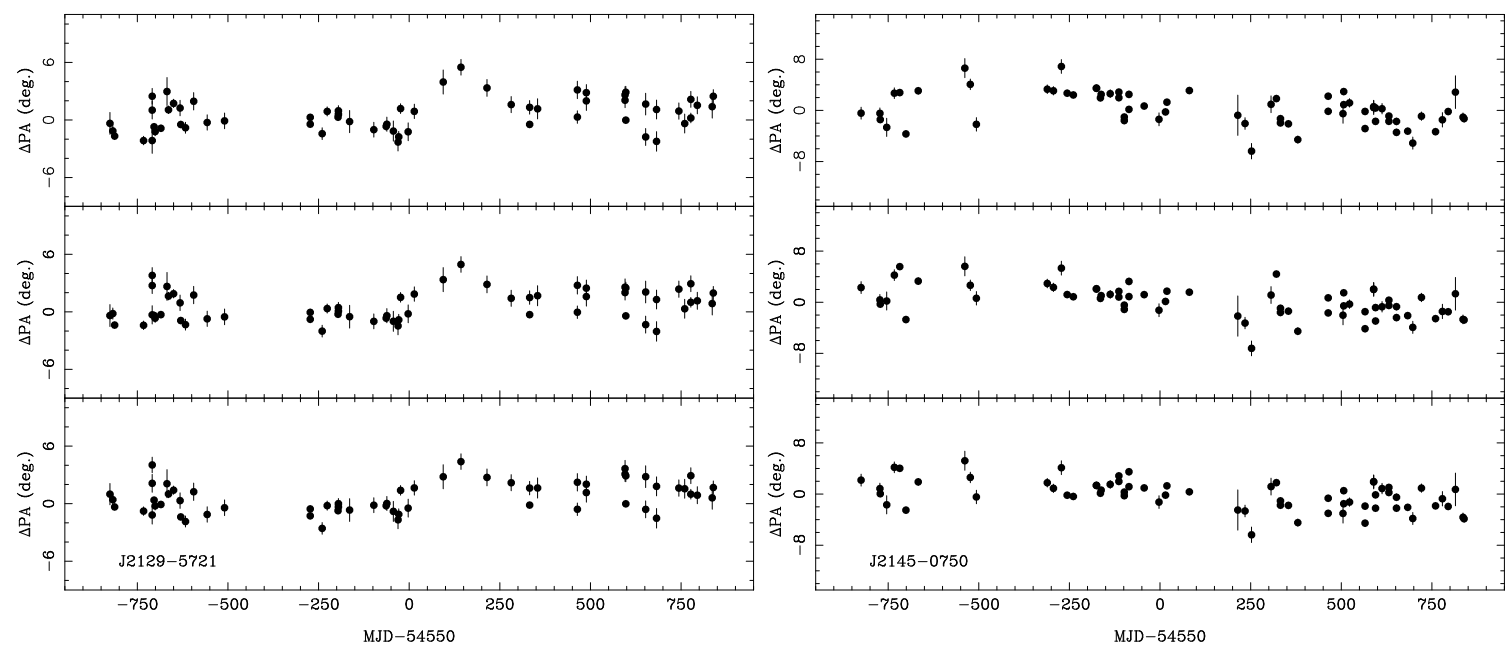

Fig. 4 PA differences as a function of time for two of the PPTA pulsars. See Figure 1 for details.

Table 2 Rms variations and reduced $\chi^{2}$ for ionosphere-corrected PAs

\begin{tabular}{ccccc}
\hline \multirow{2}{*}{ PSR } & \multicolumn{2}{c}{ FARROT } & \multicolumn{2}{c}{ IRI } \\
& $\sigma_{P A}$ & Reduced & $\sigma_{P A}$ & Reduced \\
& $\left(^{\circ}\right)$ & $\chi^{2}$ & $\left(^{\circ}\right)$ & $\chi^{2}$ \\
\hline J0437-4715 & 1.14 & 833.3 & 0.96 & 488.6 \\
J0613-0200 & 2.31 & 5.9 & 2.15 & 4.7 \\
J0711-6830 & 1.82 & 3.5 & 1.70 & 3.3 \\
J1022+1001 & 1.22 & 192.8 & 0.93 & 96.5 \\
J1024-0719 & 1.41 & 47.3 & 1.31 & 43.1 \\
& & & & \\
J1045-4509 & 1.74 & 5.6 & 1.63 & 4.6 \\
J1600-3053 & 1.33 & 10.6 & 1.08 & 6.5 \\
J1603-7202 & 1.35 & 4.0 & 1.29 & 2.9 \\
J1643-1224 & 1.43 & 5.7 & 1.09 & 3.5 \\
J1713+0747 & 1.40 & 76.9 & 1.37 & 70.7 \\
& & & & \\
J1730-2304 & 1.83 & 25.2 & 1.71 & 17.0 \\
J1732-5049 & 1.93 & 1.8 & 1.92 & 1.5 \\
J1744-1134 & 1.28 & 779.9 & 0.98 & 411.9 \\
J1824-2452 & 1.08 & 16.5 & 0.95 & 11.1 \\
J1857+0943 & 3.02 & 5.3 & 2.71 & 4.0 \\
& & & & \\
J1909-3744 & 1.15 & 138.1 & 1.11 & 123.5 \\
J1939+2134 & 1.22 & 64.3 & 1.42 & 50.6 \\
J2124-3358 & 1.44 & 3.4 & 1.31 & 3.0 \\
J2129-5721 & 1.72 & 5.4 & 1.71 & 5.2 \\
J2145-0750 & 2.49 & 31.8 & 2.31 & 20.2 \\
\hline & & & & \\
\hline
\end{tabular}


small, often $\ll 1^{\circ}$. Residual calibration errors can exceed the rms noise in the stronger pulsars, leading to PA offsets and hence scatter in the PAs. Low-level unexcised RFI can affect both the accuracy of the calibration and the Stokes data themselves also leading to PA scatter. Finally, although the ionospheric correction has reduced the short-term scatter in the PAs, it cannot be completely accurate as it relies on models for the ionosphere and its variations whereas the actual ionosphere is dynamic and changes on short timescales in often unpredictable ways. It is difficult to separate these contributions to the scatter in the corrected PAs, but it is probable that this last effect is dominant. Near real-time monitoring of the total electron content, for example, using the dual-frequency Global Positioning System (e.g., Garner et al. 2008), should help to improve the accuracy of the RMi measurements and hence reduce the overall short-term scatter in the PAs.

Figures 11-4 show that there is little if any significant long-term variation in the corrected PAs. This implies that the mean PA of the emitted pulses and hence the orientation of the spin and magnetic axes of the pulsar have varied by $\lesssim 1^{\circ}$ over the nearly 5 -year data span. This is not surprising since six of the 20 pulsars are isolated and none of the 14 binary pulsars is in a close orbit where geodetic precession might be expected (Breton et al. 2008; Manchester et al. 2010). In addition, most of the binary companions are evidently of relatively low mass $\left(\lesssim 0.5 \mathrm{M}_{\odot}\right)$. Because of the great age of these MSPs, any free precession resulting from interactions or deformations in the spin-up phase would be likely to have dissipated by now (cf. Glampedakis et al. 2009).

Figures 5 - 8 show the IRI-corrected PAs for the PPTA pulsars. The straight line on each plot results from a weighted least-squares fit of a straight line to the data. To reduce the short-term scatter, weighted averages of the corrected PAs over 100-day blocks are shown in the middle panel, again with the fitted straight line. If we assume that there has been no variation in the intrinsic PA over this interval, we can convert the observed PA variations to equivalent RM variations using Equation 1 as shown in the lower panel. Table 3 gives the slope of the fitted line and its estimated uncertainty and the corresponding RM gradient for the 20 pulsars. Only a few of these gradients are significant at more than the $3 \sigma$ level; mostly the observations are consistent with no long-term change in PA or interstellar RM within the uncertainties.

As discussed in $\$ 1$, we can use the ratio of the RM gradient to the DM gradient to estimate the line-ofsight component of the magnetic field in a putative time-varying region. DM variations for the PPTA pulsars were measured by You et al. (2007). There is only one year of DM data overlap with the PA variation data but the DM gradients given in Table 3 , obtained by fitting to this overlap year, are representative of the longer-term gradients. Because of the high-precision timing possible for these MSPs, the absolute changes in DM, while significant in some cases, are extremely small. This, combined with the fact that we effectively have just upper limits on $d \mathrm{DM} / d t$ for most of our sample, means that, for these pulsars, we can just place upper limits of several $100 \mu \mathrm{G}$ on the interstellar field strength in the time-varying region. Values and uncertainties of $\left\langle B_{\|}\right\rangle_{\text {var }}$ given in Table 3 have been computed using Equation 5 with the uncertainties in $d \mathrm{DM} / d t$ and $d \mathrm{RM} / d t$ assumed to be independent.

Apparently significant long-term PA and implied RM variations $(>3 \sigma)$ are observed for five of the pulsars: PSRs J0613-0200, J1022+1001, J1603-7202, J1909-3744 and J2145-0750. For PSRs J1022+1001 and $\mathrm{J} 2145-0750, d \mathrm{DM} / d t$ is not well determined and so the mean field is very uncertain. For PSRs J0613-0200, J1603-7202 and J1909-3744, there are significant values for both $d \mathrm{RM} / d t$ and $d \mathrm{DM} / d t$, so we can compute values for $\left\langle B_{\|}\right\rangle_{\text {var }}$ as listed in Table 3. For most of the pulsars, the $\left\langle B_{\|}\right\rangle_{\text {var }}$ values or limits are much larger than typical interstellar fields of a few $\mu \mathrm{G}$ (Han et al. 2006) and even the field expected in magnetic filaments associated with supernova remnants (Hamilton et al. 1985; Rankin et al. 1988).

For PSRs J1024-0719, J1045-4509 and J1824-4509 the upper limits on $\left\langle B_{\|}\right\rangle_{\text {var }}$ are interestingly low, of order $10 \mu \mathrm{G}$. This is comparable to the estimated interstellar total field strength, including both largescale regular fields and small-scale irregular fields (Beuermann et al. 1985; Han et al. 2004). These results show that variations in the interstellar RM for these pulsars are not dominated by any strong filament along the line of sight.

As well as the significant long-term variations in RM discussed above, Figures [5 - 8 show that there are apparently significant fluctuations in the smoothed RM variations on a timescale of a few hundred days for some of the MSPs. For PSR J1939+2134, the PA appears to increase at a rate of about $3 \mathrm{rad} \mathrm{m}^{-2} \mathrm{yr}^{-1}$ for the first 150 days (MJD $53900-54050$ ). Over this same interval, the DM is decreasing at about $-8 \times 10^{-4} \mathrm{~cm}^{-3} \mathrm{pc} \mathrm{yr}^{-1}$ (You et al. 2007). If both these variations are occurring in a single region, they imply a line-of-sight magnetic field of about $4.5 \mathrm{mG}$ in the region. Although such fields are observed close to stars, such an intersection is highly improbable (except for the Sun - discussed below). Most probably, the observed variations are not common to a single region, but represent statistical fluctuations in the accumulated RM and DM over 

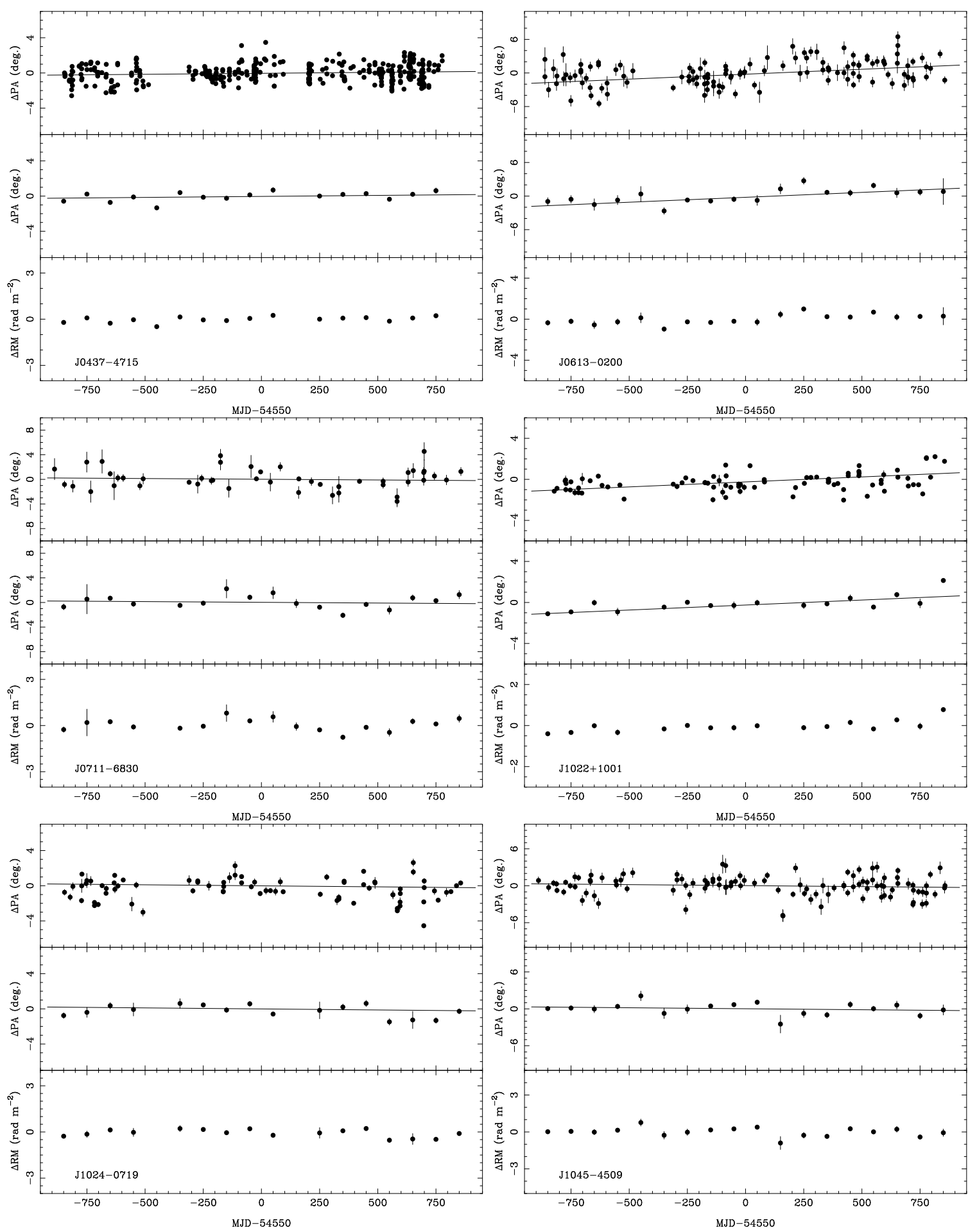

Fig. 5 PA variations for six of the PPTA pulsars. The upper panel of each sub-figure has the IRI-corrected PA variations showing the fitted linear trend. The middle panel shows the same data averaged over 100-day blocks with the fitted line as in the upper panel. The lower panel shows the smoothed variations converted to RM changes using Equation (1). 

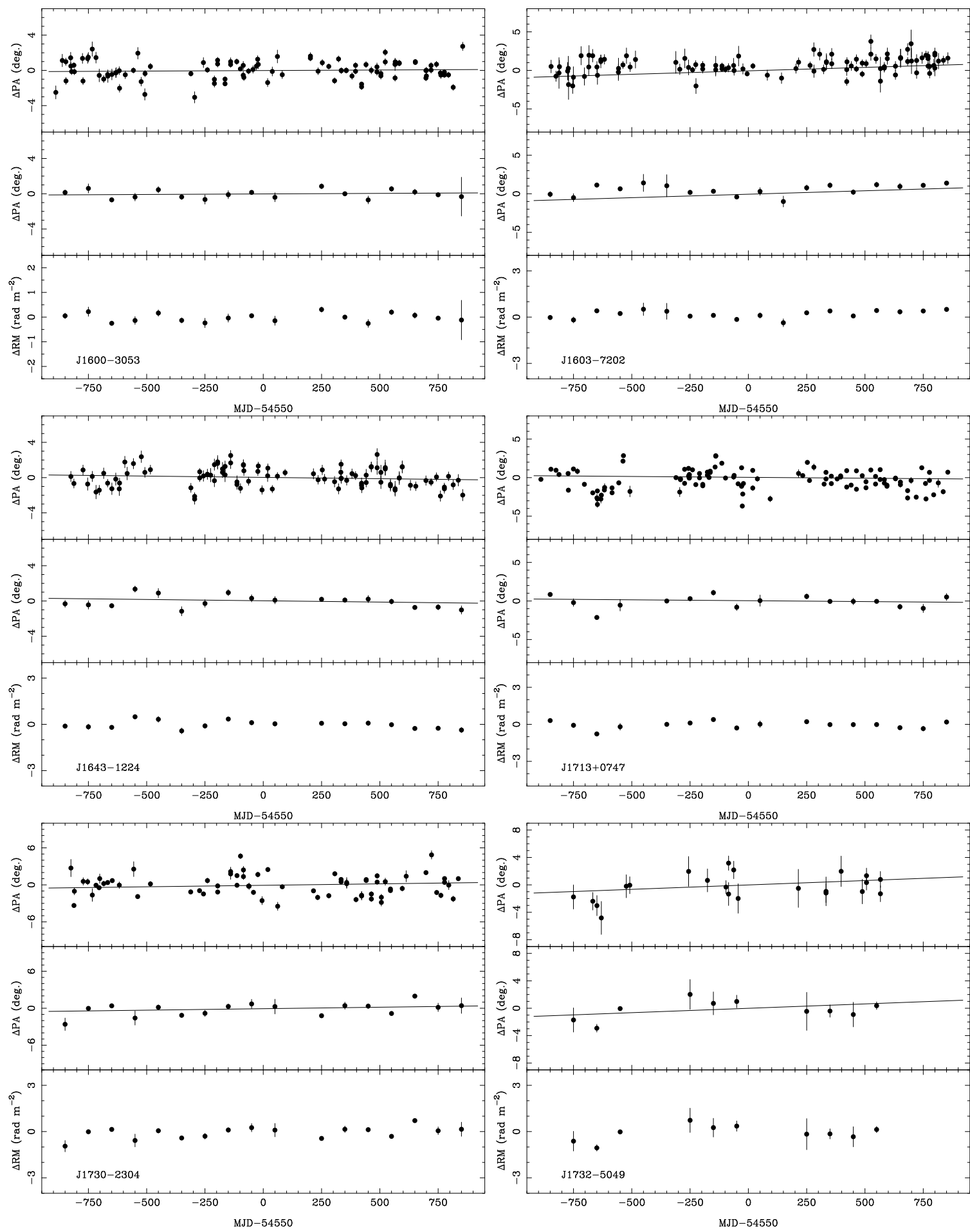

Fig. 6 PA variations for six of the PPTA pulsars. See Figure 5 for details. 

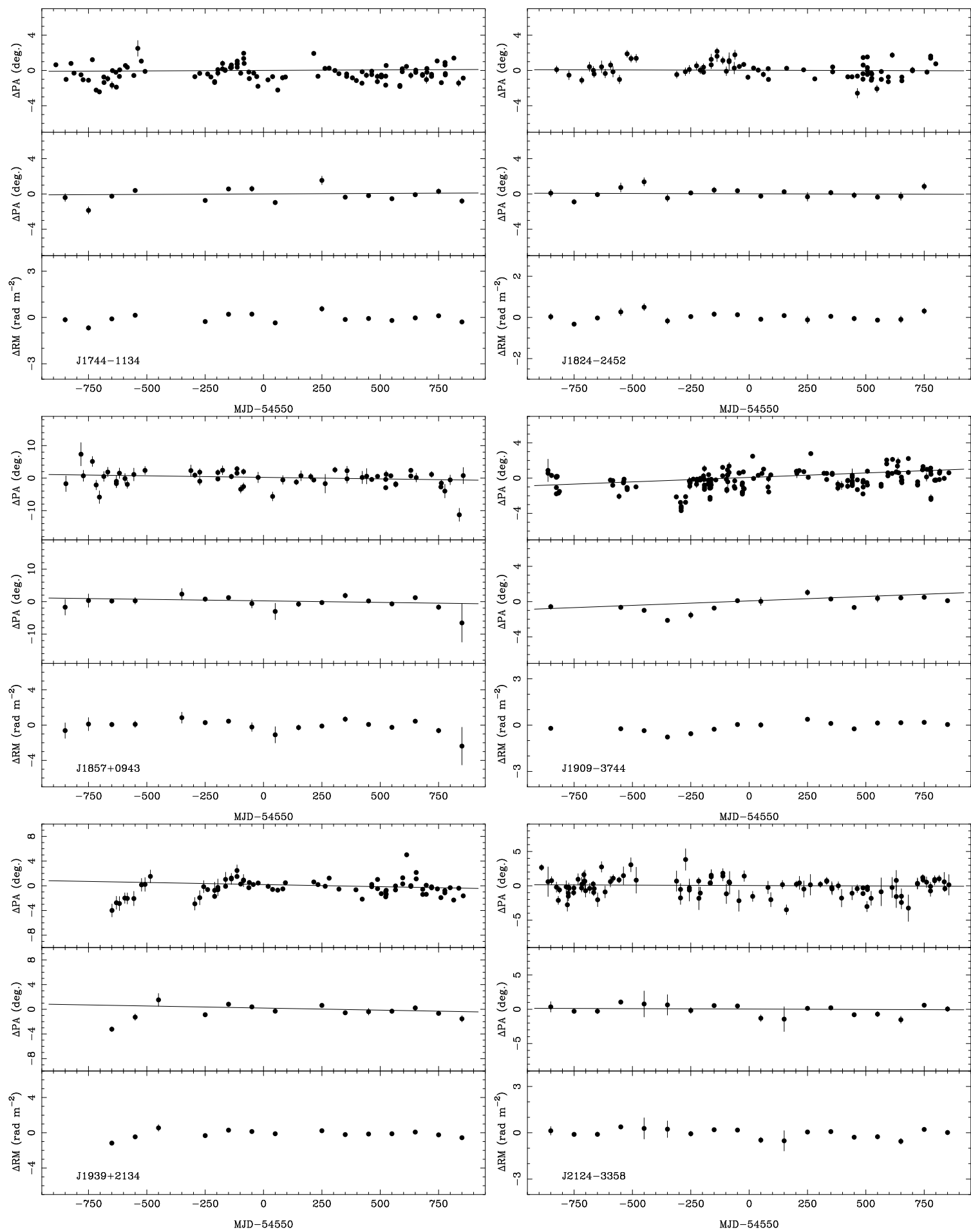

Fig. 7 PA variations for six of the PPTA pulsars. See Figure 5 for details. 

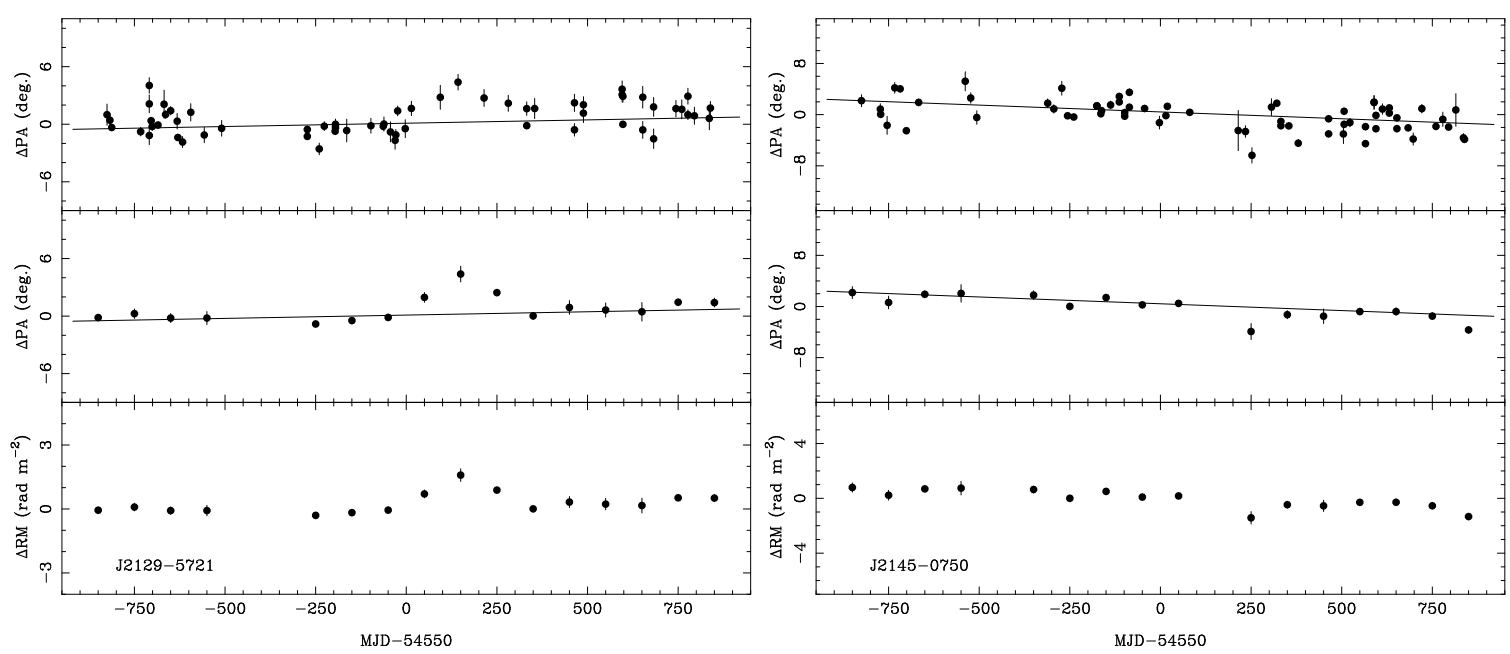

Fig. 8 PA variations for two of the PPTA pulsars. See Figure 5 for details.

Table 3 PA gradients, implied RM gradients, DM gradients and apparent line-ofsight magnetic field in the time-varying region for the PPTA pulsars.

\begin{tabular}{|c|c|c|c|c|}
\hline PSR & $\begin{array}{l}d \mathrm{PA} / d t \\
\left({ }^{\circ} \mathrm{yr}^{-1}\right)\end{array}$ & 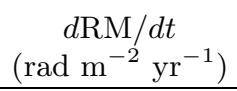 & $\begin{array}{c}d \mathrm{DM} / d t \\
\left(\mathrm{~cm}^{-3} \mathrm{pc} \mathrm{yr}^{-1}\right)\end{array}$ & $\begin{array}{c}\left\langle B_{\|}\right\rangle_{\text {var }} \\
(\mu \mathrm{G})\end{array}$ \\
\hline J0437-4715 & $0.08 \pm 0.03$ & $0.03 \pm 0.01$ & $(3 \pm 3) \times 10^{-5}$ & $1200 \pm 1300$ \\
\hline J0613-0200 & $0.64 \pm 0.13$ & $0.23 \pm 0.05$ & $(4.3 \pm 1.6) \times 10^{-4}$ & $660 \pm 280$ \\
\hline J0711-6830 & $-0.08 \pm 0.13$ & $-0.03 \pm 0.05$ & $(-5.3 \pm 7.8) \times 10^{-4}$ & $70 \pm 160$ \\
\hline $\mathrm{J} 1022+1001$ & $0.35 \pm 0.06$ & $0.13 \pm 0.02$ & $(-2.0 \pm 5.5) \times 10^{-4}$ & $-800 \pm 2200$ \\
\hline J1024-0719 & $-0.08 \pm 0.09$ & $-0.03 \pm 0.03$ & $(4.8 \pm 1.9) \times 10^{-3}$ & $-8 \pm 8$ \\
\hline J1045-4509 & $-0.12 \pm 0.09$ & $-0.04 \pm 0.03$ & $(-1.03 \pm 0.01) \times 10^{-2}$ & $5 \pm 4$ \\
\hline $\mathrm{J} 1600-3053$ & $0.05 \pm 0.07$ & $0.02 \pm 0.02$ & $(-9 \pm 10) \times 10^{-4}$ & $-30 \pm 40$ \\
\hline $\mathrm{J} 1603-7202$ & $0.33 \pm 0.08$ & $0.12 \pm 0.03$ & $(3.4 \pm 0.8) \times 10^{-3}$ & $40 \pm 10$ \\
\hline $\mathrm{J} 1643-1224$ & $-0.11 \pm 0.08$ & $-0.04 \pm 0.03$ & $(-3.2 \pm 3.7) \times 10^{-4}$ & $150 \pm 210$ \\
\hline $\mathrm{J} 1713+0747$ & $-0.08 \pm 0.08$ & $-0.03 \pm 0.03$ & $(-6 \pm 24) \times 10^{-5}$ & $620 \pm 2500$ \\
\hline J1730-2304 & $0.17 \pm 0.12$ & $0.06 \pm 0.05$ & $(-2.3 \pm 7.1) \times 10^{-4}$ & $-320 \pm 1000$ \\
\hline J1732-5049 & $0.47 \pm 0.30$ & $0.17 \pm 0.11$ & $(7 \pm 11) \times 10^{-4}$ & $300 \pm 510$ \\
\hline $\mathrm{J} 1744-1134$ & $0.04 \pm 0.06$ & $0.01 \pm 0.02$ & $(-5.0 \pm 8.7) \times 10^{-4}$ & $-20 \pm 70$ \\
\hline $\mathrm{J} 1824-2452$ & $-0.02 \pm 0.10$ & $-0.01 \pm 0.04$ & $(6.2 \pm 0.7) \times 10^{-3}$ & $-2 \pm 8$ \\
\hline $\mathrm{J} 1857+0943$ & $-0.35 \pm 0.21$ & $-0.13 \pm 0.08$ & $(-1.4 \pm 3.3) \times 10^{-3}$ & $110 \pm 280$ \\
\hline J1909-3744 & $0.37 \pm 0.07$ & $0.13 \pm 0.02$ & $(-4.6 \pm 0.9) \times 10^{-4}$ & $-350 \pm 90$ \\
\hline $\mathrm{J} 1939+2134$ & $-0.25 \pm 0.11$ & $-0.09 \pm 0.04$ & $(3.3 \pm 1.7) \times 10^{-4}$ & $-340 \pm 230$ \\
\hline $\mathrm{J} 2124-3358$ & $-0.04 \pm 0.07$ & $-0.02 \pm 0.02$ & $(-4.5 \pm 8.1) \times 10^{-4}$ & $50 \pm 110$ \\
\hline J2129-5721 & $0.25 \pm 0.10$ & $0.09 \pm 0.04$ & $(5 \pm 4) \times 10^{-4}$ & $220 \pm 200$ \\
\hline $\mathrm{J} 2145-0750$ & $-0.78 \pm 0.14$ & $-0.28 \pm 0.05$ & $(3.5 \pm 4.6) \times 10^{-4}$ & $-990 \pm 1300$ \\
\hline
\end{tabular}


the whole path resulting from random and uncorrelated spatial and temporal variations in the interstellar electron density and magnetic field.

It is important to note that the observed RM variations would be expected to be less than the DM variations since the RM is a vector sum (Equation (2)) whereas the DM is a scalar sum (Equation (3)). Variations in regions of oppositely directed field will cancel in RM but not in DM. A more detailed examination of the origin of these variations would require realistic modelling of the electron density and magnetic field fluctuations along representative Galactic paths. This is left for future work.

It is also worth noting that the aliased diurnal variations in ionospheric RM are approximately covariant with annual variations in RM and DM resulting from the changing path through the solar corona as the Earth moves around the Sun. Both have maxima when the pulsar transits near local noon. At least for paths that do not pass too close to the Sun, the DM variations are roughly sinusoidal; TEMPO2 has a correction term for delays occurring in the solar corona (Edwards et al. 2006; You et al. 2007a). Predicted RM variations are more complex because of structure in the solar magnetic field (Ord et al. 2007). Some of the PPTA pulsars (notably PSRs J1022+1001 and J1730-2304) have low ecliptic latitudes, but the few observations when these pulsars were close to the Sun have been excluded from the present analysis. The fact that little or no annual PA variation is evident in Figures $5-8$ shows that any variations due to the changing path through the solar corona are below the level of detectabilty for these observations of the PPTA pulsars.

The main goals of the PPTA and other similar PTA projects depend on precise measurement of pulse times of arrival (ToAs). In principle, the ionospheric RM should be taken into account when summing the data in frequency. Failure to do so would result in some depolarization of the signal. Depending on the ToA algorithm used and the details of the profile polarization this could affect the ToAs. Fortunately, at the frequencies of $1.4 \mathrm{GHz}$ and above commonly used for PTA observations, the effect is very small, with the depolarization being $\lesssim 1$ per cent across the band. Some ToA algorithms, for example, matrix template matching (van Straten 2006), solve for the PA rotation across the band and so the effect is decoupled from the ToA measurement. This is potentially important for pulsar timing at low radio frequencies.

\section{Conclusions}

The regular observations of 20 MSPs undertaken as part of the PPTA project provide an excellent data base for investigation of long-term variations in the properties of the observed pulses. In this paper we have analysed the time variations of mean absolute pulse position angle over 4.8 years. The largest systematic effect found was that due to variations in the Earth's ionosphere. Two ionospheric models, FARROT and the International Reference Ionosphere (IRI) model, were used to predict the variations in the ionospheric component, $\mathrm{RMi}$, and to compensate the observed PA changes for ionospheric variations. It was found that the IRI model gave better results than FARROT.

After compensation, the rms fluctuation in the PAs was about $1^{\circ}$ with little or no significant long-term variations over the data span for most pulsars. This implies not only that the polarization of the emitted radiation was very stable, but also that the net long-term variations in interstellar RM along the path to the pulsar were also very small, typically less than $0.1 \mathrm{rad} \mathrm{m}^{-2}$ $\mathrm{yr}^{-1}$ in absolute value. The observed variations were dominated by short-term fluctuations that most likely originate from residual calibration errors or errors in the RMi due to unmodelled short-timescale variations in the ionospheric total electron content.

Apparently significant $\mathrm{PA} / \mathrm{RM}$ variations on timescale of a few 100 days or longer were observed in some of the pulsars. Comparison with the DM variations published by You et al. (2007) showed that these RM variations imply high values of magnetic field if they are interpreted as correlated variations occurring in a small part of the path. More likely, they represent statistical fluctuations in the whole-of-path RM resulting from random spatial and temporal variations in both the interstellar electron density and magnetic field.

Acknowledgements This work was supported by NSFC project 10673021, the Knowledge Innovation Programme of the Chinese Academy of Sciences, grant no. KJCX2-YW-T09, National Basic Research Programme of China (973 Programme 2009CB824800), the Program of the Light in China's Western Region (LCWR) under grant XBBS201022 and the Key Laboratory of Radio Astronomy, Chinese Academy of Sciences. We thank Andrew Gray and Ken Tapping of the Dominion Radio Astrophysical Observatory for making the Penticton ionospheric modelling program FARROT available to us. The Parkes radio telescope is part of the Australia Telescope, which is funded by the Commonwealth of Australia for operation as a National Facility managed by the Commonwealth Scientific and Industrial Research Organisation. 


\section{References}

Beuermann K., Kanbach G., Berkhuijsen E. M., 1985, A\&A, 153, 17

Breton R. P., Kaspi V. M., Kramer M., McLaughlin M. A., Lyutikov M., Ransom S. R., Stairs I. H., Ferdman R. D., Camilo F., 2008, Science, 321, 104

Edwards R. T., Hobbs G. B., Manchester R. N., 2006, MNRAS, 372, 1549

Garner T. W., Gaussiran II T. L., Tolman B. W., Harris R. B., Calfas R. S., Gallagher H., 2008, Adv. Space Res., 42,720

Glampedakis K., Andersson N., Jones D. I., 2009, MNRAS, 394, 1908

Hamilton P. A., Hall P. J., Costa M. E., 1985, MNRAS, $214,5 \mathrm{P}$

Hamilton P. A., McCulloch P. M., Manchester R. N., Ables J. G., Komesaroff M. M., 1977, Nature, 265, 224

Han J.-L., Ferrière K., Manchester R. N., 2004, ApJ, 610 , 820

Han J. L., Manchester R. N., Lyne A. G., Qiao G. J., van Straten W., 2006, ApJ, 642, 868

Hobbs G. B., Edwards R. T., Manchester R. N., 2006, MNRAS, 369, 655

Hotan A. W., van Straten W., Manchester R. N., 2004, PASA, 21, 302

Johnston S., Ball L., Wang N., Manchester R. N., 2005, MNRAS, 358, 1069

Manchester R. N., 1974, ApJ, 188, 637

Manchester R. N., 2008, in Bassa C., Wang Z., Cumming A., Kaspi V. M., eds, 40 Years of Pulsars: Millisecond Pulsars, Magnetars and More Vol. 983, The Parkes Pulsar Timing Array Project. American Institute of Physics, New York, pp 584-592

Manchester R. N., Kramer M., Stairs I. H., Burgay M., Camilo F., Hobbs G. B., Lorimer D. R., Lyne A. G., McLaughlin M. A., McPhee C. A., Possenti A., Reynolds J. E., van Straten W., 2010, ApJ, 710, 1694

Noutsos A., Johnston S., Kramer M., Karastergiou A., 2008, MNRAS, 386, 1881

Ord S. M., Johnston S., Sarkissian J., 2007, Solar Phys., 245, 109

Rankin J. M., Campbell D. B., Isaacman R. B., Payne R. R., 1988, A\&A, 202, 166

Staveley-Smith L., Wilson W. E., Bird T. S., Disney M. J., Ekers R. D., Freeman K. C., Haynes R. F., Sinclair M. W., Vaile R. A., Webster R. L., Wright A. E., 1996, PASA, 13,243

van Ommen T. D., D'Alesssandro F. D., Hamilton P. A., McCulloch P. M., 1997, MNRAS, 287, 307

van Straten W., 2004, ApJS, 152, 129

van Straten W., 2006, ApJ, 642, 1004

van Straten W., Manchester R. N., Johnston S., Reynolds J. E., 2010, PASA, 27, 104

Verbiest J. P. W., Bailes M., Bhat N. D. R., Burke-Spolaor S., Champion D. J., Coles W., Hobbs G. B., Hotan A. W., Jenet F., Khoo J., et al. 2010, Class. Quantum Grav., 27, 084015

Weisberg J. M., Cordes J. M., Kuan B., Devine K. E., Green J. T., Backer D. C., 2004, ApJS, 150, 317
Yan W., Manchester R. N., van Straten W., Reynolds J., Hobbs G., Wang N., Bailes M., Bhat R., Burke-Spolaor S., Champion D., Coles W., Hotan A., Khoo J., Oslowski S., Sarkissian J., Verbiest J., Yardley D., 2011, MNRAS, in press

You X. P., Hobbs G., Coles W. A., Manchester R. N., Edwards R., Bailes M., Sarkissian J., Verbiest J. P. W., van Straten W., Hotan A., Ord S., Jenet F., Bhat N. D. R., Teoh A., 2007, MNRAS, 378, 493

You X. P., Hobbs G. B., Coles W. A., Manchester R. N., Han J. L., 2007a, ApJ, 671, 907 